\title{
CUIDADOS PALIATIVOS NO ÂMBITO HOSPITALAR EM PACIENTES COM INSUFICIÊNCIA CARDÍACA
}

\section{Laurynês de Castro' ${ }^{1}$, João Paulo Coelho Cintra', Nathalia Antunes dos Santos ${ }^{1}$, Pedro Henrique Falcuci $^{1}$, Sarah da Silva Candido ${ }^{2}$ and Cynthia Kallás Bachur ${ }^{3}$}

\author{
1Universidade de Franca (UNIFRAN), Franca - SP - Brazil \\ ${ }^{2}$ Escola de Enfermagem de Ribeirão Preto- USP - Ribeirão Preto - SP - Brazil \\ ${ }^{3}$ Docente do Curso de Fisioterapia e de Medicina da Universidade de Franca (UNIFRAN) \\ -Franca - SP. -Brazil
}

\section{ARTICLE INFO}

Article History:

Received $15^{\text {th }}$ December, 2020

Received in revised form

$27^{\text {th }}$ January, 2021

Accepted $20^{\text {th }}$ February, 2021

Published online $15^{\text {th }}$ March, 2021

Key Words:

Insuficiência Cardíaca; Cuidados Paliativos; Qualidade de Vida; Assistência Terminal.

*Corresponding author: Laurynês de Castro

\begin{abstract}
Objetivo: Identificar pacientes com IC que necessitem de cuidados paliativos, a partir da Escala de Performance Paliativa (PPS) e Escala de avaliação de Sintomas de Edmonton (ESAS). Métodos: Trata-se de um estudo exploratório, descritivo e transversal, realizado em um hospital público de uma cidade no interior do estado de São Paulo em 2019. A população alvo foi os pacientes com IC, amostra por conveniência, e os critérios de inclusão foram de acordo com a classe funcional III/IV, e idade maior que 50 anos. Para coleta de dados, foi adotado um questionário com identificação e dados da doença, a escala PPS para avaliar a funcionalidade dos participantes, e a ESAS para avaliar os sintomas. Resultados: Participaram 47 pacientes,idade $71,04 \pm 10,58$ anos. Observou-se que $38,3 \%$ se enquadravam nos critérios mais prevalentes para necessidade de cuidados paliativos, sendo $66,7 \%$ do sexo feminino, uma média de idade total de 74,2 anos $\pm 10,22$, cerca de 3,9 internações durante o curso da doença e das etiologias identificadas a mais frequente é a chagásica com 27,7\%. Conclusão: A partir dos resultados obtidos, percebeu-se que há necessidade de rastrear, a partir das escalas utilizadas, os pacientes que necessitam de cuidados paliativos como parte do tratamento.
\end{abstract}

Copyright (C) 2021, Laurynês de Castro et al. This is an open access article distributed under the Creative Commons Attribution License, which permits unrestricted use, distribution, and reproduction in any medium, provided the original work is properly cited.

Citation: Laurynês de Castro, João Paulo Coelho Cintra, Nathalia Antunes dos Santos, Pedro Henrique Falcuci, Sarah da Silva Candido and Cynthia Kallás Bachur. 2021. "Cuidados paliativos no âmbito hospitalar em pacientes com insuficiência cardíaca”, International Journal of Development Research, $11,(03), 45114-45119$.

\section{INTRODUÇÃO}

A Insuficiência Cardíaca (IC) é uma síndrome complexa, em que o coração é incapaz de bombear sangue devido a desordens funcionais e estruturais, que levam ao risco do desenvolvimento de sintomas de um baixo débito cardíaco e/ou congestão pulmonar ou sistêmica (Sociedade Brasileira de Cardiologia, 2018; Porth, 2010). Pode ser classificada quanto a fração de ejeção do ventrículo esquerdo, de acordo com a gravidade e a progressão da doença (Sociedade Brasileira de Cardiologia, 2018). Apresenta incidência e prevalência elevada em todo mundo, e estima-se que a doença atinge mais de 23 milhões de pessoas. No Brasil existem aproximadamente 6 milhões de pacientes, sendo diagnosticados 240 mil pacientes anualmente (Teixeira, 2015). De acordo com as Diretrizes Brasileiras de Insuficiência Cardíaca (2018), a prevalência da doença tende a aumentar com a idade, sendo $1 \%$ naqueles com idade variando entre 55 a 64 anos, e 17,4\% naqueles maiores que 85 anos (Sociedade Brasileira de Cardiologia, 2018). Esses números estão em ascensão provavelmente devido ao aumento da expectativa de vida. A causa mais frequente de internações por doenças do aparelho circulatório é a IC em indivíduos com mais de 20 anos de idade no Brasil, sendo responsável por 3\% das internações gerais e $23 \%$ das internações relacionadas ao aparelho cardiovascular (Corrêa, 2015), no ano de 2012 somente com hospitalizações no sistema público, foram gastos 356,9 milhões de reais ${ }^{1}$, segundo dados atuais do DATASUS houve aproximadamente 217 mil internações de outubro de 2017 a outubro de 2018 (Datasus, 2019). Além disso, dados demonstram que apenas no ano de 2012, houve 26.694 óbitos por IC no Brasil (Albuquerque, 2015). O diagnóstico precoce e o tratamento efetivo diminuem a morbimortalidade e os custos do tratamento. Depois da utilização de todo o arsenal terapêutico, uma notável quantidade de pacientes com IC progridem para o estágio da doença e ficam sem a possibilidade de transplante cardíaco ou dispositivo de suporte circulatório, o que os leva a alternativa dos 
cuidados paliativos, que segundo a Organização Mundial da Saúde (OMS) visa à melhora da qualidade de vida do enfermo e de seus familiares, prevenindo e aliviando o sofrimento por meio da identificação precoce, avaliação eficaz e tratamento rigoroso da dor e outros problemas físicos, psíquicos, sociais e espirituais (Academia Nacional de Cuidados Paliativos, 2012). De fato, o cuidado paliativo é uma modalidade de cuidado ativo, que enfatiza a atenção integral ao paciente portador de doença limitadora da vida, que é definida como o conjunto das patologias, para as quais não existe tratamento curativo eficaz disponível (Knobel, 2016). O paciente portador de IC que podem ser admitidos para cuidados paliativos tem algumas características mais prevalentes que incluem: mais que uma internação ou descompensação nos últimos seis meses; classe funcional IV segundo o NYHA (Fauci, 2016) que é caracterizado por incapacidade de realizar qualquer atividade sem apresentar desconforto e presença de sintomas no repouso; qualidade de vida ruim e dependências nas atividades de vida diária; transplante cardíaco ou suporte circulatório descartados; caquexia cardíaca ou baixa albumina sérica; e diagnóstico de paciente terminal, que é definido quando o princípio da não maleficência predomina sobre o da beneficência (Sociedade Brasileira de Cardiologia, 2018). Como forma de facilitar a detecção e a resolução dos sinais e sintomas que são encontrados nos pacientes que necessitam de cuidados paliativos foram criadas diversas escalas, entre elas a Escala de Avaliação de Sintomas de Edmonton (ESAS), criada em 1991 no Hospital Geral de Edmonton no Canadá, desenvolvida para ser utilizada diariamente na avaliação dos sintomas. No Brasil, a ESAS foi traduzida e no ano de 2009 (Monteiro, 2009). Outro instrumento que também se destaca na literatura é a Escala de Performance Paliativa, no inglês Palliative Performace Scale (PPS), criado em 1996 pelo Victoria Hospice no Canadá, amplamente utilizado e validado no cenário nacional em medicina paliativa (Academia Nacional de Cuidados Paliativos, 2012). Trata-se de uma ferramenta que permite acompanhar a curva evolutiva da doença e avaliar a funcionalidade. Ela pode ser útil na tomada de decisão, previsão de diagnóstico e definição de terminalidade (Ho, 2008). A PPS oferece condições de diagnosticar a perspectiva de cura, facilitando a suspensão de terapias desnecessárias e por vezes prejudiciais (Chan, 2012).

Com base nos dados relevantes na literatura, acredita-se que identificar os pacientes que necessitam de cuidados paliativos, pode auxiliar no prognóstico da doença e auxiliar a equipe profissional a planejar condutas cabíveis e o momento adequado de iniciar este recurso, podendo evitar procedimentos de alta complexidade e garantir uma menor exposição aos riscos decorrentes de procedimentos invasivos. Sendo assim, o objetivo deste estudo foi identificar os pacientes que necessitem de cuidados paliativos em um hospital público; avaliar a funcionalidade dos pacientes a partir da escala de PPS; avaliar os sintomas dos pacientes a partir da Escala de Avaliação de Sintomas de Edmonton e capacitar os profissionais da saúde do hospital, onde foi desenvolvido este estudo, quanto as orientações básicas dos Cuidados Paliativos.

\section{MÉTODOS}

Trata-se de um estudo exploratório, descritivo e transversal, realizado em um hospital público de uma cidade no interior do estado de São Paulo, durante o ano de 2019. A amostra foi por conveniência, composta por pacientes internados com diagnóstico de ICC, voluntários, sem distinção de gênero ou etnia.

\section{Instrumentos de coleta de dados}

- Identificação dos participantes:aplicou-se um questionário com os seguintes dados: nome, gênero, idade, estado civil, responsável/cuidador, com quem mora, religião/crença, o que sabe sobre a sua doença, cronologia da doença atual e tratamento realizado (época do diagnóstico, tratamentos realizados, quantidade de hospitalizações e descompensações). $\mathrm{O}$ instrumento foi elaborado e aplicado pelos pesquisadores deste estudo em todos os participantes. A escolha do instrumento se justifica, porque a coleta pode se dar com pessoas alfabetizadas ou analfabetas, de forma que os pesquisadores estabeleçam um padrão de coleta de dados de forma a facilitar a tabulação e garantir credibilidade.

- Escala de Performance Paliativa (PPS) (Chan, 2012): esta escala é utilizada para avaliar a funcionalidade dos pacientes. Possui 11 níveis, de 0 a 100 , divididos em intervalo de 10 , avaliando 5 aspectos: capacidade de locomoção, atividade e evidência da doença, capacidade para o autocuidado, ingesta alimentar espontânea e nível de consciência. A leitura se faz da esquerda para a direita e, quanto mais baixo o valor, maior a intensidade dos parâmetros à direita.

- Escala de Avaliação de Sintomas de Edmonton (Monteiro, 2009) (ESAS): esta escala é utilizada para avaliar os sintomas dos pacientes, especificamente: dispneia, dor, apetite, fadiga, náuseas, depressão, sonolência, ansiedade e mal-estar. Esse método de avaliação consiste em escalas visuais e numéricas que variam de zero a dez, sendo 0 ausência de sintoma e $10 \mathrm{com}$ maior intensidade.

- A partir dos resultados obtidos no presente estudo, foi proposto aos profissionais da saúde vinculados ao hospital, juntamente com a aprovação da diretoria, a realização de 3 seminários,sobre cuidados paliativos, a partir aulas expositivas com duração de 40 minutos, com apresentação de slides em Power Point, em datas previamente estabelecidas e de comum acordo entre os pesquisadores e os funcionários, para que não ocorra prejuízo aos horários, realizada no próprio ambiente de trabalho.

- Durante o seminário foi abordado os resultados do trabalho, como a quantidade de pacientes com IC que necessitam de cuidados paliativos, o perfil do paciente terminal (qual sexo, idade mais prevalentes), assim como as estatísticas dos principais sintomas dos pacientes analisados. $\mathrm{Na}$ apresentação, propôs-se o ideal manejo a esse tipo de paciente, mostrando a partir da literatura, o melhor manejo dos sintomas identificados como mais prevalentes, e exemplos de criações de unidades de Cuidados Paliativos e suas consequências (como melhor qualidade de vida ao doente, menor tempo de hospitalização, menor custo diário ao hospital e educação da equipe para identificar um paciente que se encaixa nesse tipo de cuidado, entre outros).

- Como critério de inclusão, considerou-se idade acima de 65 anos, diagnóstico de ICC em classe funcional III e IV. E como critério de exclusão arecusa em participar do projeto.

- Todas as informações obtidas no instrumento aplicado foram armazenadas no software Microsoft Excel. Em seguida, foram transferidas para o programa estatístico STATA 9.0 para cálculos de frequência absoluta e relativa. A análise descritiva foi expressa a partir de média e desvio padrão.

\section{RESULTADOS}

Participaram do estudo 47 pacientes com diagnóstico de ICC internados em um hospital público, sendo 22 (46,8\%) mulheres e 25 $(53,2 \%)$ homens, com idade média de $71,05 \pm 9,49$ e $71,04 \pm 11,84$ anos respectivamente. A distribuição das características sócio demográficas de todos os participantes estão descritas na Tabela 1.

A Tabela 2 apresenta a distribuição das características da doença, da internação, e do tratamento realizado entre os pacientes diagnosticados com ICC. Com o propósito de avaliar a funcionalidade dos pacientes diagnosticados com ICC, os dados foram analisados a partir do score final da "Escala de Performance Paliativa". A Tabela 3 apresenta a distribuição dos valores identificados. Do total dos pacientes avaliados a partir da PPS, apenas $1(4 \%)$ do sexo masculino apresentou pontuação de $100 \%$ da escala, o qual configura atividade funcional completa e favorável nos domínios: deambulação, atividade e evidência de doença, autocuidado, ingestão e nível de consciência. De acordo com os valores identificados, 12 (25,5\%) pacientes foram classificados no Nível I ( 80 a 100 pontos): indivíduo apto para as atividades normais e o trabalho, sem que seja necessário qualquer 
Tabela 1. Distribuição das características sócio demográficas dos pacientes diagnosticados com ICC internados em um hospital público. Franca, 2019 (n=47)

\begin{tabular}{|c|c|c|c|c|c|c|}
\hline \multicolumn{7}{|c|}{ Sexo } \\
\hline Variáveis & \multicolumn{2}{|c|}{$\begin{array}{l}\text { Feminino } \\
\mathrm{n}=22(46,8 \%)\end{array}$} & \multicolumn{2}{|c|}{$\begin{array}{l}\text { Masculino } \\
\mathrm{n}=25(53,2 \%)\end{array}$} & \multicolumn{2}{|c|}{ Total } \\
\hline Estado Civil & $\mathrm{n}$ & $\%$ & $\mathrm{n}$ & $\%$ & $\mathrm{n}$ & $\%$ \\
\hline Solteiro & 1 & 4,5 & 4 & 16,0 & 5 & 10,6 \\
\hline Casado ou em relacionamento estável & 8 & 36,3 & 14 & 56,0 & 22 & 46,8 \\
\hline Separado ou divorciado & 1 & 4,5 & 3 & 12,0 & 4 & 8,5 \\
\hline Viúvo & 9 & 40,9 & 4 & 16,0 & 13 & 27,6 \\
\hline \multicolumn{7}{|l|}{ Responsável ou cuidador } \\
\hline Sim & 14 & 63,6 & 12 & 54,5 & 26 & 55,3 \\
\hline Não & 8 & 36,3 & 13 & 59,0 & 21 & 44,6 \\
\hline \multicolumn{7}{|l|}{ Moradores da casa } \\
\hline 1 a 2 & 10 & 45,4 & 11 & 44,0 & 21 & 44,6 \\
\hline 3 a 4 & 8 & 36,3 & 11 & 44,0 & 19 & 40,4 \\
\hline 5 a 6 & 1 & 4,5 & 1 & 4,0 & 2 & 4,2 \\
\hline$>7$ & 3 & 13,6 & 2 & 8,0 & 5 & 10,6 \\
\hline \multicolumn{7}{|l|}{ Religião/crença } \\
\hline Católica & 10 & 45,4 & 11 & 44,0 & 21 & 44,6 \\
\hline Judaica & 0 & 0,0 & 0 & 0,0 & 0 & 0,0 \\
\hline Evangélica & 11 & 50,0 & 9 & 36,0 & 20 & 42,5 \\
\hline Espírita & 0 & 0,0 & 2 & 8,0 & 2 & 4,2 \\
\hline Outra & 1 & 4,5 & 1 & 4,0 & 2 & 4,2 \\
\hline Sem religião & 0 & 0,0 & 2 & 8,0 & 2 & 4,2 \\
\hline
\end{tabular}

A Tabela 2 apresenta a distribuição das características da doença, da internação, e do tratamento realizado entre os pacientes diagnosticados com ICC.

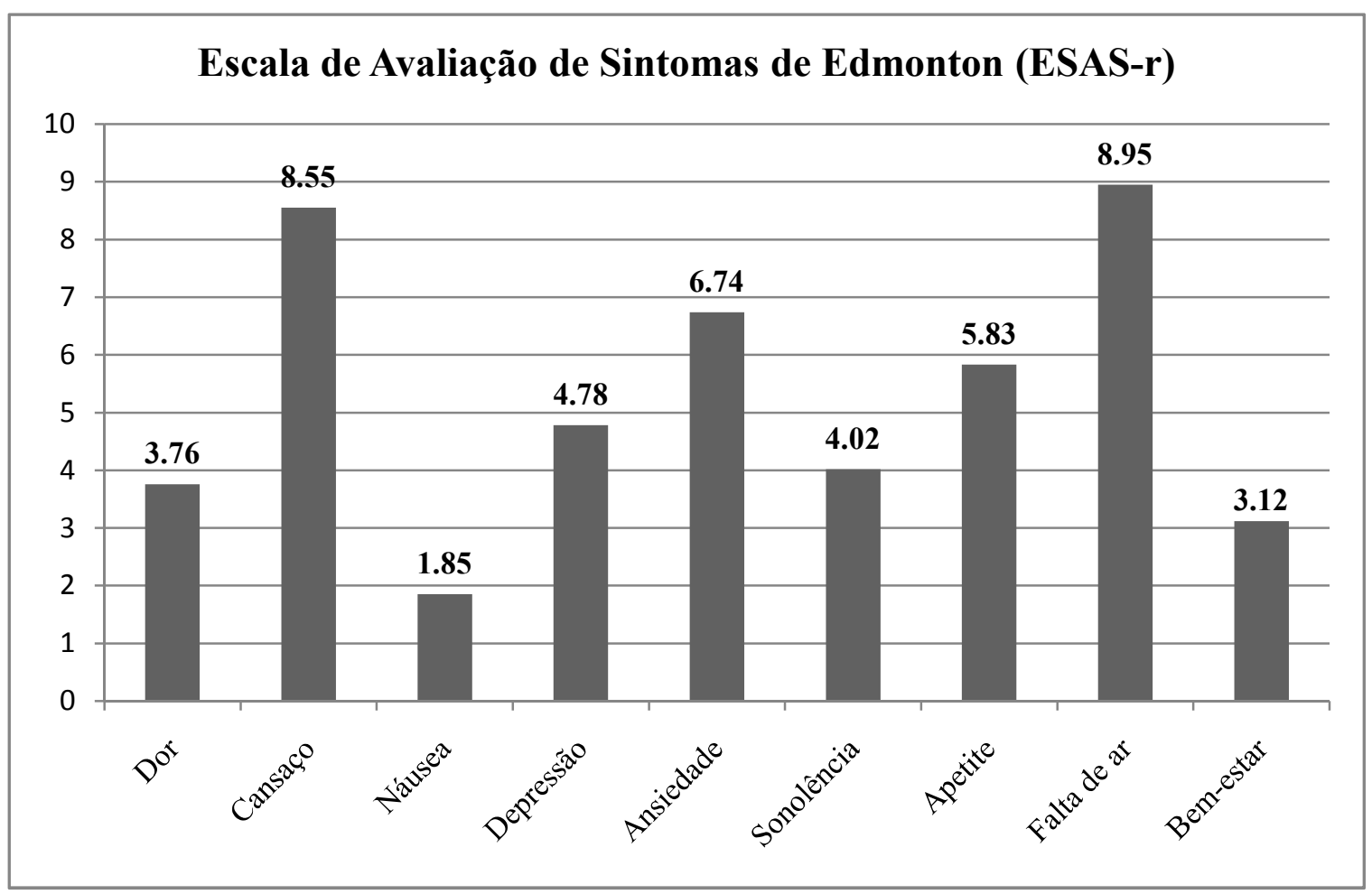

Figura 1. Análise descritiva dos sintomas avaliados pela "Escala de Avaliação de Sintomas de Edmonton" em pacientes diagnosticados com ICC internados em um hospital público. Franca, 2019 (n=47)

cuidado em especial; 28 (59,5\%) pacientes no Nivel II (50 a 70 pontos): indivíduo inapto para o trabalho, mas apto para viver em casa e cuidar de muitas de suas necessidades, exigindo assistência e suporte necessários bastante variáveis; 7 (14,8\%) no Nível III (0 a 40 pontos): indivíduo inapto para cuidar de si mesmo, requerendo cuidados hospitalares ou especializados, com a doença progredindo rapidamente. Em qualquer nível de classificação do paciente de acordo com a escala PPS, é exigida a necessidade do gerenciamento intensivo dos sintomas e das demandas que o paciente e a família apresentam. Para a identificação dos sintomas físicos e psicológicos dos pacientes avaliados, os dados foram analisados a partir dos valores apresentados na aplicação da "Escala de Avaliação de Sintomas de Edmonton”. A Figura 1 apresenta a análise descritiva dos sintomas listados.
A partir da análise dos dados presentes na Escala de Avaliação de Sintomas de Edmonton, foi possível identificar que o sintoma mais referido com pontuação máxima foi a "Falta de ar" relatado por 29 (61,7\%) pacientes, seguido por "Cansaço" 27 (57,4\%) e "Ansiedade $14(29,7 \%)$. O sintoma menos relatado foi a "Náusea", de acordo com a representação de $31(65,9 \%)$ pacientes com pontuação " 0 ". Observou-se que 38,3\% se enquadram nos critérios mais prevalente para necessidade de cuidados paliativos que são, dependência funcional (PPS $<50 \%$ ), associada a um dos seguintes parâmetros, presença de sintomas ao repouso (queixa com nota 9 ou 10, no ESAS); mais de uma internação ou descompensação, nos últimos 6 meses. Foi encontrado que $66,7 \%$ é do sexo feminino, sendo a média de idade delas de $75 \pm 7,68$ anos, com relação as internações a média foi 4,6 $\pm 6,5$ internações durante o curso da doença. 
Tabela 2. Distribuição das características da doença, da internação e do tratamento realizado entre pacientes diagnosticados com ICC internados em um hospital público. Franca, 2019 (n=47)

\begin{tabular}{|c|c|c|c|c|c|c|}
\hline \multirow[b]{3}{*}{ Época do diagnóstico de IC } & \multicolumn{6}{|c|}{ Sexo } \\
\hline & \multicolumn{2}{|c|}{$\begin{array}{l}\text { Feminino } \\
n=22(46,8 \%)\end{array}$} & \multicolumn{2}{|c|}{$\begin{array}{l}\text { Masculino } \\
\qquad \mathrm{n}=25(53,2 \%)\end{array}$} & \multicolumn{2}{|c|}{ Total } \\
\hline & $\mathrm{n}$ & $\%$ & $\mathrm{n}$ & $\%$ & $\mathrm{n}$ & $\%$ \\
\hline Há menos de 1 ano & 5 & 22,7 & 10 & 40,0 & 15 & 31,9 \\
\hline $1-5$ anos & 7 & 31,8 & 8 & 32,0 & 15 & 31,9 \\
\hline $6-10$ anos & 2 & 9,1 & 2 & 8,0 & 4 & 8,5 \\
\hline Mais de 10 anos & 6 & 27,2 & 5 & 20,0 & 11 & 23,4 \\
\hline Não sabe / Não Lembra & 2 & 9,1 & 0 & 0,0 & 2 & 4,2 \\
\hline \multicolumn{7}{|l|}{ Tratamentos realizados } \\
\hline \multicolumn{7}{|l|}{ Beta Bloqueador (BB) } \\
\hline Sim & 19 & 86,3 & 18 & 72,0 & 37 & 78,7 \\
\hline Não & 3 & 13,6 & 5 & 20,0 & 8 & 17,0 \\
\hline Não identificada & 0 & 0,0 & 2 & 8,0 & 2 & 4,2 \\
\hline \multicolumn{7}{|c|}{ Diurético Poupador de Potássio (DPK) } \\
\hline Sim & 13 & 59,0 & 11 & 44,0 & 24 & 51,0 \\
\hline Não & 9 & 40 & 12 & 48,0 & 21 & 44,6 \\
\hline Não identificada & 0 & 0 & 2 & 8,0 & 2 & 4,2 \\
\hline \multicolumn{7}{|c|}{ Inibidor da Enzima Conversora de Angiotensina (IECA) } \\
\hline Sim & 8 & 36,3 & 10 & 40,0 & 18 & 38,2 \\
\hline Não & 14 & 63,6 & 13 & 52,0 & 27 & 7,4 \\
\hline Não identificada & 0 & 0,0 & 2 & 8,0 & 2 & 4,2 \\
\hline \multicolumn{7}{|c|}{ Bloqueador do Receptor de Angiotensina (BRA) } \\
\hline $\operatorname{Sim}$ & 5 & 22,7 & 2 & 8,0 & 7 & 14,8 \\
\hline Não & 17 & 77,2 & 21 & 84,0 & 38 & 80,8 \\
\hline Não identificada & 0 & 0,0 & 2 & 8,0 & 2 & 4,2 \\
\hline \multicolumn{7}{|l|}{ Digitálicos } \\
\hline Sim & 1 & 4,5 & 0 & 0,0 & 1 & 2,1 \\
\hline Não & 21 & 95,4 & 23 & 92,0 & 44 & 93,6 \\
\hline Não identificada & 0 & 0,0 & 2 & 8,0 & 2 & 4,2 \\
\hline \multicolumn{7}{|l|}{ Diurético de Alça (DA) } \\
\hline Sim & 21 & 95,4 & 22 & 88,0 & 43 & 91,4 \\
\hline Não & 1 & 4,54 & 1 & 4,0 & 2 & 4,2 \\
\hline Não identificada & 0 & 0,0 & 2 & 8,0 & 2 & 4,2 \\
\hline \multicolumn{7}{|l|}{ Antiarrítmico } \\
\hline $\operatorname{Sim}$ & 3 & 13,6 & 4 & 16,0 & 7 & 14,9 \\
\hline Não & 19 & 86,3 & 19 & 76,0 & 38 & 80,8 \\
\hline Não identificada & 0 & 0,0 & 2 & 8,0 & 2 & 4,2 \\
\hline Bloqueador de Canal de Cálc & & & & & & \\
\hline $\operatorname{Sim}$ & 7 & 31,8 & 5 & 20,0 & 12 & 25,0 \\
\hline Não & 15 & 68,1 & 18 & 72,0 & 33 & 70,2 \\
\hline Não identificada & 0 & 0,0 & 2 & 8,0 & 2 & 4,2 \\
\hline Dobutamina & & & & & & \\
\hline Sim & 1 & 4,5 & 3 & 12,0 & 4 & 8,5 \\
\hline Não & 21 & 95,4 & 20 & 80,0 & 41 & 87,2 \\
\hline Não identificada & 0 & 0,0 & 2 & 8,0 & 2 & 4,2 \\
\hline Cateterismo & & & & & & \\
\hline Sim & 6 & 27,2 & 10 & 40,0 & 16 & 34,0 \\
\hline Não & 16 & 72,7 & 13 & 52,0 & 29 & 61,7 \\
\hline Não identificada & 0 & 0,0 & 2 & 8,0 & 2 & 4,2 \\
\hline Exacerbações e internações & & & & & & \\
\hline 1 a 3 & 16 & 72,7 & 21 & 85,0 & 37 & 78,7 \\
\hline 4 a 6 & 4 & 18,1 & 1 & 4,0 & 5 & 10,6 \\
\hline$\geq 7$ & 2 & 9,0 & 3 & 12,0 & 5 & 10,6 \\
\hline Fração de Ejeção & & & & & & \\
\hline Preservada $(>50 \%)$ & 5 & 22,7 & 2 & 8,0 & 7 & 14,8 \\
\hline Intermediária (40 - 49\%) & 2 & 9,0 & 3 & 12,0 & 5 & 10,6 \\
\hline Reduzida $(<40 \%)$ & 4 & 18,1 & 8 & 32,0 & 12 & 25,5 \\
\hline Não identificada & 11 & 50,0 & 12 & 48,0 & 23 & 48,9 \\
\hline Etiologia & & & & & & \\
\hline Isquêmica & 5 & 22,7 & 9 & 36,0 & 14 & 29,7 \\
\hline Hipertensiva & 1 & 4,5 & 2 & 8,0 & 3 & 6,3 \\
\hline Chagásica & 6 & 27,2 & 3 & 12,0 & 9 & 19,1 \\
\hline Valvar & 1 & 4,5 & 1 & 4,0 & 2 & 4,2 \\
\hline Outras & 2 & 9,0 & 3 & 12,0 & 5 & 10,6 \\
\hline Não identificada & 7 & 31,8 & 7 & 28,0 & 14 & 29,7 \\
\hline
\end{tabular}

Com o propósito de avaliar a funcionalidade dos pacientes diagnosticados com ICC, os dados foram analisados a partir do score final da "Escala de Performance Paliativa". A Tabela 3 apresenta a distribuição dos valores identificados.

Nos 33, 3\% do sexo masculino a média encontrada foi $72,66 \pm 14,8$ anos e de internações foi $2,5 \pm 2,3$. A etiologia identificada mais associada a esse quadro é a chagásica na totalidade dos pacientes, com $27,7 \%$.

\section{DISCUSSÃo}

Observa-se na literatura que a IC aumenta sua prevalência com a idade o que foi verificado na amostra cuja média de idade é de 71 anos para ambos os sexos. Comparado a um estudo de 82 pessoas 
Tabela 3. Distribuição dos valores avaliados pela "Escala de Performance Paliativa" em pacientes diagnosticados com ICC internados em um hospital público. Franca, 2019 (n=47)

\begin{tabular}{|c|c|c|c|c|c|c|c|c|}
\hline & $\begin{array}{l}\text { PP* } \\
(\%)\end{array}$ & Deambulação & $\begin{array}{l}\text { Atividade e evidência da } \\
\text { doença }\end{array}$ & Autocuidado & Ingestão & $\begin{array}{l}\text { Nível } \\
\text { de consciência }\end{array}$ & $\mathrm{n}$ & $\%$ \\
\hline \multirow[b]{3}{*}{$\frac{\bar{d}}{\mathrm{D}}$} & 100 & Completa & $\begin{array}{l}\text { Normal, sem evidência de } \\
\text { doença }\end{array}$ & Completo & Normal & Completa & 1 & 2,12 \\
\hline & 90 & Completa & $\begin{array}{l}\text { Normal, alguma evidência de } \\
\text { doença }\end{array}$ & Completo & Normal & Completa & 0 & 0,0 \\
\hline & 80 & Completa & $\begin{array}{l}\text { Com esforço, alguma } \\
\text { evidência de doença }\end{array}$ & Completo & Normal & Completa & 11 & 23,4 \\
\hline \multirow{3}{*}{ 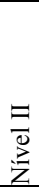 } & 70 & Reduzida & $\begin{array}{l}\text { Incapaz para o trabalho, } \\
\text { alguma evidência de doença }\end{array}$ & Completo & Normal ou reduzida & Completa & 10 & 21,2 \\
\hline & 60 & Reduzida & $\begin{array}{l}\text { Incapaz de realizar hobbies, } \\
\text { doença significativa }\end{array}$ & Assistência ocasional & Normal ou reduzida & $\begin{array}{l}\text { Períodos de confusão } \\
\text { ou completa }\end{array}$ & 8 & 17,0 \\
\hline & 50 & $\begin{array}{l}\text { Sentado } \\
\text { ou deitado }\end{array}$ & $\begin{array}{l}\text { Incapacitado para qualquer } \\
\text { trabalho, doença extensa }\end{array}$ & Assistência considerável & Normal ou reduzida & $\begin{array}{l}\text { Períodos de confusão } \\
\text { ou completa }\end{array}$ & 10 & 21,2 \\
\hline \multirow{5}{*}{$\begin{array}{l}\text { 三 } \\
\stackrel{\Xi}{\mathrm{Z}}\end{array}$} & 40 & Acamado & $\begin{array}{l}\text { Incapacitado para qualquer } \\
\text { trabalho, doença extensa }\end{array}$ & Assistência quase completa & Normal ou reduzida & $\begin{array}{l}\text { Períodos de confusão } \\
\text { ou completa }\end{array}$ & 3 & 6,38 \\
\hline & 30 & Acamado & $\begin{array}{l}\text { Incapacitado para qualquer } \\
\text { trabalho, doença extensa }\end{array}$ & Dependência completa & Reduzida & $\begin{array}{l}\text { Períodos de confusão } \\
\text { ou completa }\end{array}$ & 2 & 4,25 \\
\hline & 20 & Acamado & $\begin{array}{l}\text { Incapacitado para qualquer } \\
\text { trabalho, doença extensa }\end{array}$ & Dependência completa & $\begin{array}{l}\text { Ingestão } \\
\text { limitada a colheradas }\end{array}$ & $\begin{array}{l}\text { Períodos de confusão } \\
\text { ou completa }\end{array}$ & 1 & 2,12 \\
\hline & 10 & Acamado & $\begin{array}{l}\text { Incapacitado para qualquer } \\
\text { trabalho, doença extensa }\end{array}$ & Dependência completa & Cuidados com a boca & Confuso ou em coma & 1 & 2,12 \\
\hline & 0 & Morte & - & - & - & - & 0 & 0 \\
\hline
\end{tabular}

*PP: Escala de Performance Paliativa

Figura 2. Medidas paliativas no paciente com IC.

\begin{tabular}{|c|c|}
\hline Sintomas & Cuidados paliativos \\
\hline Dispneia e fadiga & $\begin{array}{l}\text { Inotrópicos positivos, furosemida em doses liberais, oxigenoterapia em altasdoses, morfina, benzodiazepínicos, reabilitação e } \\
\text { ventiladores no ambiente }\end{array}$ \\
\hline Tosse & Avaliar congestão e rever uso de inibidores da enzima conversora de angiotensina \\
\hline Depressão & $\begin{array}{l}\text { Inibidores seletivos da receptação da serotonina, evitar antidepressivos tricíclicos, psicoterapia, reabilitação, terapia } \\
\text { comportamental e suporte emocional }\end{array}$ \\
\hline Ansiedade & Benzodiazepínicos, psicoterapia, exercícios respiratórios e técnicas de relaxamento \\
\hline Dor & $\begin{array}{l}\text { Aplicar escalas de dor, evitar anti-inflamatórios não hormonais, usar morfina, psicoterapia e terapia ocupacional. Se } \\
\text { relacionada ao cardioversão desfibrilador implantável, considerar ajustes }\end{array}$ \\
\hline Tontura & Ajustar doses de fármacos hipotensores \\
\hline Edema & Esquemas de diurético em infusão domiciliar, otimização terapêutica para IC e cuidados com a pele \\
\hline Náuseas e vômitos & $\begin{array}{l}\text { Avaliar suspensão de ácido acetilsalicílico, considerar uso de agentes pró cinéticos (metoclopramida e ondansetrona), } \\
\text { haloperidol e refeições de pequenos volumes em intervalos menores }\end{array}$ \\
\hline Constipaçãointestinal & Laxantes, flexibilizar a restrição hídrica se possível e adequar dieta para anticonstipante \\
\hline Insônia & $\begin{array}{l}\text { Avaliar e tratar depressão, ansiedade, nictúria, apneia do sono, delirium concomitantes, promover técnicas de higiene do } \\
\text { sono }\end{array}$ \\
\hline Delirium & $\begin{array}{l}\text { Avaliar causas clínicas precipitantes, como distúrbios metabólicos, descompensação cardiovascular, infecção ou efeito } \\
\text { adverso de medicação. Minimizar fármacos anticolinérgicos. Baixa dose de antipsicótico, se o sintoma causar risco para o } \\
\text { paciente ou seu cuidador. }\end{array}$ \\
\hline
\end{tabular}

com pacientes em IC em estágio avançado, foi visto que o perfil epidemiológico é similar apresentando uma média de 68 anos. Além desse parâmetro foi encontrado outras semelhanças como a predominância do sexo masculino de $51 \%$, casados $52,4 \%$ que vão de encontro com os resultados encontrados que são $53 \%$ e $46,8 \%$,respectivamente (Orzechowski, 2019). Poff et al observaram em seus estudos com uma amostra de 724 pacientes com IC NYHA III/IV apresenta uma idade média de 66,1 anos com superioridade numérica dos homens de 52\% (Poffo, 2017). A IC pode ser classificada quanto a fração de ejeção podendo ser preservada, intermediária ou reduzida.No presente estudo foi encontrado que as mulheres apresentam fração de ejeção preservada em sua maioria, sendo semelhante em uma revisão de literatura realizado com pacientes tendo a prevalência maior mulheres e idosos, estando associada a múltiplas comorbidades (Mesquita, 2010). De acordo Diretriz Brasileira de Insuficiência Cardíaca, as etiologias mais frequentes da IC são isquêmica, hipertensiva, chagásica e valvar (Sociedade Brasileira de Cardiologia, 2018), e no presente estudo, a etiologia mais frequente são isquemia miocárdica $(29,7 \%)$, chagásica $(19,1 \%)$, hipertensiva $(6,3 \%)$ e valvar $(4,2 \%)$, entre outras causas e não identificadas $(40,3 \%)$. No estudo com 148 pessoas foram encontradas como etiologia prevalente a isquêmica (20,3\%), sucedida pelo valvar $(16,4 \%)$ chagásica (14\%),hipertensiva $(11,8 \%)$ e as idiopáticas $(37,5 \%)$ (Viana, 2018). O tratamento medicamentoso utilizado para os pacientes com IC sintomática inclui uma terapia tripla usual com Inibidor da Enzima Conversora de Angiotensina (IECA) ou Bloqueador do Receptor de Angiotensina 2 (BRA), associado a betabloqueador e diurético poupador de potássio(DPK). Além disso, podem ser utilizados adjuvantes dos tratamentos como diuréticos de alça, bloqueador do canal de cálcio (BCC), digitálicos, dobutamina entre outros (Sociedade Brasileira de Cardiologia, 2018). Em uma revisão de literatura foi evidenciado que o uso de betabloqueador reduz o efeito prejudicial da estimulação simpática, aumentando a sobrevida. Os IECAs e BRA bloqueiam os efeitos deletérios do sistema renina-angiotensina-aldosterona o que limita o remodelamento cardíaco. Os DPK são eficazes na redução da mortalidade e número de internações em pacientes com NYHA III/IV e fração de ejeção $<35 \%$, sendo esses medicamentos classe $1 \mathrm{em}$ nível de evidência A (Mizzaci 2017). Nesse trabalho foram encontrados resultados aproximados ao discutido anteriormente, com a utilização de betabloqueador em $78,7 \%$, IECA ou BRA em $53 \%$ e DPK $51 \%$. O diurético de alça é um fármaco utilizado para sintomas relacionados a congestão (edema, crepitação pulmonar, turgência de jugular e dispneia), como a maioria dos pacientes em classe NYHA III/IV apresentam esses sintomas, quase a totalidade da amostra $(91,4 \%)$ faz uso dessa medicação (Medeiros, 2014). Em uma revisão de literatura foi visto que $90 \%$ dos pacientes analisados a partir da escala PPS, apresentam 50\% de sobrevida em apenas seis meses, e pacientes com PPS de $20 \%$ indicam sinais de terminalidade (Vasconcelos, 2018). 
De acordo com os dados colhidos $36 \%$ dos pacientes da amostra tem uma sobrevida em média de seis meses. No mesmo estudo citado anteriormente também foi analisado o nível da PPS nos pacientes com ICC e em comparação das medianas dos resultados encontramos $80 \%$ no seguinte estudo e $60 \%$ nos dados colhidos no presente estudo. Os cuidados paliativos visam melhorar as condições de vida dos enfermos e seus familiares, e isso inclui o controle dos sintomas físicos, psíquicos e espirituais que trazem o sofrimento do paciente. A escala ESAS é um instrumento que permite a avaliação e observação de nove sintomas físicos e psicológicas em pacientes em cuidados paliativos. As queixas mais prevalentes analisadas no presente estudo foram a falta de ar $(8,95)$, cansaço $(8,55)$ e ansiedade $(6,74)$. A Figura 2 sugere medidas paliativas adicionais a otimização terapêutica habitual em paciente com IC. A Diretriz Brasileira de IC traz que os pacientes em estágio $\mathrm{D}$ da doença,que alcançam a intratabilidade clínica, necessitam de transplante cardíaco, o qual muitas vezes não é possível, o que leva a possibilidade de cuidados paliativos. Algumas características encontradas no paciente com IC levam a indicação desse tipo de tratamento, entre elas estão sintomas ao repouso, ou classe IV no NYHA, dependência para atividades diárias, diagnóstico de terminalidade, falha de dispositivo de suporte circulatório, internações e descompensações repetidas nos últimos seis meses ${ }^{(1)}$. A partir dos resultados do presente estudo, $38,3 \%$ dos pacientes alcançam o perfil de necessidade de cuidados paliativos, entre esses a característica mais prevalente está no sexo feminino, por volta 75 anos, e 4 a 5 internações desde o diagnóstico da doença. Os resultados apresentados no presente estudo apontam que a maioria dos pacientes acometidos com ICC avançada são do sexo masculino, apresentam uma idade média de 70 anos, sendo a maioria de etiologia não definida, seguida da isquêmica.

\section{CONCLUSÃO}

De acordo com os resultados do presente estudo, observa-se que há a necessidade de rastrear a classificação da IC, segundo NHYA III/IV, para a indicação de cuidados paliativos,a partir das escalas PPS e ESAS para identificar sintomas associados. Torna-se imprescindível o reconhecimento dos profissionais da saúde para o manejo da doença, e se possível, uma capacitação periódica para tal fim.

\section{REFERÊNCIAS}

Academia Nacional de Cuidados Paliativos. Manual de cuidados paliativos. Rio de Janeiro: Diagraphic, 2012.

Albuquerque, DC., Neto, JDS., Bacal, F., Rohde, LEP., et al. 2015. I Registro Brasileiro de insuficiência cardíaca - aspectos clínicos, qualidade assistencial e desfechos hospitalares. Rio de Janeiro, Sociedade Brasileira de Cardiologia.

Chan E-Y, Wu H-Y, Chan Y-H. 2012. Revisiting the palliative performance scale: Change in scores during disease trajectory predicts survival. Palliat Med., 27(4):367-74.
Corrêa, AG; Makdisse, M; Katz, M; Santana, TC; Yokota, PKO; Galvão, TFG; Bacal, F. 2015. Análise de diretriz de tratamento versus protocolo assistencial em 'pacientes internados por insuficiência cardíaca. São Paulo, Sociedade Brasileira de Cardiologia.

Datasus. 2019. Departamento de informática do Sistema Único de Saúde. [Citado em 2019 Jan 14] Disponível em: tabnet.datasus.gov.br/cgi/deftohtm.exe?cih/cnv/sxuf.def

Fauci, AS. et al. 2016. Harrison Medicina Interna. 19ed. Rio de Janeiro, Mc Graw Hill.

Ho F, Lau F, Downing MG, Lesperance M. 2008. A reliability and validity study of the palliative performance scale. BMC Palliative Care. 7:10.

Knobel, E. 2016. Condutas no paciente grave. 4. ed. São Paulo: Editora Atheneu.

Medeiros RBd. 2014. Aplicação sequencial da escala de performance paliativa em pacientes paliativos internados em um hospital geral. (Monografia para graduação); Repositório UFSC; Florianópolis.

Mesquita ET, Jorge AJL. Tratamento da Insuficiência Cardíaca com Fração de Ejeção Normal. Arquivos Brasileiros Cardiol, 2010. Vol.94;3.

Mizzaci CC, Rieira R, Martimbianco ALC. 2017. Tratamento farmacológico para insuficiênciacardíaca sistólica crônica e as evidências disponíveis: uma revisão narrativa da literatura. Diagnóstico e Tratamento. 22 (1):8-20

Monteiro DR. 2009. Escala de Edmonton e Cuidados Paliativos: Revisão Integrativa. Porto Alegre, UFRGS - Revista gaúcha de enfermagem. Vol. 31; 4.

Orzechowski R, et al., 2019. Necessidade de cuidados paliativos em pacientes com insuficiência cardíaca avançada internados em um hospital terciário. Revista da escola de enfermagem da USP, .vol.53.

Poffo MR, et al., 2017. Perfil dos pacientes internados por insuficiencia cardíaca em hospital terciário. International Journal of Cardiovascular Sciences. Janeiro: p. 189-198.

Porth, CM; Matfin, G. 2010. Fisiopatologia. $8^{\mathrm{a}}$ ed. Rio de Janeiro: Guanabara Koogan.

Sociedade Brasileira de Cardiologia. 2018. Diretriz Brasileira de Insuficiência Cardíaca Crônica e Aguda. Arquivos Brasileiros Cardiol, $\mathrm{n}^{\circ} 111$; 436-539.

Teixeira, TG; Xavier, LEF; Rosa, LA; Fonseca, CBR; Carvalho, VT. 2015. Cuidados paliativos no manejo da insuficiência cardíaca. Minas Gerais, Revista Medicina Minas Gerais,: ${ }^{\circ}$ 5; S14-S17.

Vasconcelos GB, Pereira PM. 2018. Cuidados Paliativos em atenção domiciliar: uma revisão bibliográfica. Revista de Administração em saúde, $18 ; \mathrm{N}^{\circ} 70$.

Viana PADS, et al., 2018. Perfil de pacientes internados para tratamento de insuficiencia cardíaca descompesada. SANARE. 2018; 17; n.01,p.15-23. 